\title{
Caspase-Dependent Apoptosis
}

National Cancer Institute

\section{Source}

National Cancer Institute. Caspase-Dependent Apoptosis. NCI Thesaurus. Code C42785.

Any apoptotic process that involves pathways that activate intracellular proteolytic caspases. 